\title{
Bioactive Glass Coatings Synthesized by Pulsed Laser Deposition Technique
}

\author{
J. Kwiatkowska*, K. SuchaneK and B. Rajchel \\ The Henryk Niewodniczański Institute of Nuclear Physics, Polish Academy of Sciences \\ E. Radzikowskiego 152, 31-342 Kraków, Poland
}

\begin{abstract}
Surface modification of medical implants is often required to improve their biocompatibility or, through bioactive properties of the surface material, facilitate its intergrowth with the living tissue. Bioactive-glass coatings can serve that purpose for the bone implants. We report a successful preparation of silicate-phosphate bioactive-glass coating on titanium substrate using the pulsed laser deposition method and present the coating characterization in terms of bonding configuration and chemical activity. The former was studied with high-resolution Raman microspectroscopy and revealed the presence of structural units responsible for the material's bioactivity. The bioactivity was also tested directly, in vitro, by soaking the samples in the simulated body fluid and examining the result with the Raman spectroscopy. The Raman spectrum, typical of hydroxyapatite was observed proving that the bone-like-material formed on the coating's surface.
\end{abstract}

PACS: 61.43.Fs, 63.50.Lm, 78.30.Ly, 81.15.Fg, 87.85.J-

\section{Introduction}

Bioactive glasses are a promising material for implant technology due to their ability to react with physiological environment and to form an interface layer of a bone-like material bonding them strongly, in vivo, to the bone tissue [1-4]. This interesting feature of the bioglasses results from the presence of glass-network modifiers in their structure, which through necessity of maintaining charge balance break the integrity of the silicate network and form weak bonds with terminal oxygens. The network fragmentation enables partial dissolution of the glass in the physiological fluids, which initiates a series of reactions at the glass surface.

The network of the glass constitute the $\mathrm{SiO}_{4}$ tetrahedra linked together at corners by bridging oxygens $(\mathrm{BO})$. The modifiers like $\mathrm{Na}_{2} \mathrm{O}, \mathrm{CaO}$, and $\mathrm{K}_{2} \mathrm{O}$ break the connections and their cations bond to the corner oxygens which no longer bridge two neighbouring Si atoms and are thus called non-bridging oxygens, NBOs. The amount and arrangement of the NBOs determine the bioactivity of the glasses.

The silicate network is commonly described in terms of $Q^{n}(\mathrm{Si})$ structural units (see e.g. [5]), where $Q$ denotes the tetrahedral $\mathrm{SiO}_{4}$ unit and $n$ is the number of bridging oxygens per tetrahedron, and thus $Q^{4}$ represents fully polymerized $\mathrm{SiO}_{4}$ network, $Q^{3}, Q^{2}, Q^{1}$ units have respectively $3,2,1$ bridging oxygens and $Q^{0}$ denotes isolated $\mathrm{SiO}_{4}$ units with no bridging oxygen atoms. The presence

* corresponding author; e-mail: Jadwiga.Kwiatkowska@ifj.edu.pl and abundance of particular $Q^{n}$ units give the picture of the network's connectivity and can be determined with high-resolution Raman spectroscopy.

Bulk bioglasses, despite their biocompatible and bioactive properties cannot always be used as implants as they are too brittle and exhibit low mechanical strength. To overcome this drawback bioactive-glass coatings on other, stronger materials are fabricated. The issues to be dealt with in this case become: the preserving of the bioactive qualities of the bulk glass in the glass coating and ensuring of good adherence of the deposited layer to the substrate. These can be achieved by the right choice of the method and deposition parameters. Pulsed laser deposition (PLD) is one of the techniques feasible to produce adherent and uniform films with controlled stoichiometry $[3,6-8]$.

The studied material is a modification of the bioglass $45 \mathrm{~S} 5$ first developed by Hench [9] and contains $\mathrm{K}_{2} \mathrm{O}$ in the formula. The high-resolution Raman spectra for the glass of this composition, not published before as far as we are aware, are presented. The analysis of bonding configuration crucial for the material's bioactivity was carried out for both the target and the PLD-formed coating. The instrument resolution was sufficient for the $Q^{n}$ components to be resolved and their contributions determined.

This paper reports the formation and characterization of silicate-phosphate bioactive-glass coating and presents the results of in vitro bioactivity test.

\section{Materials and methods}

PLD method was used to form bioactive coating on the Ti substrate. In the PLD process a high power pulsed 
laser beam is used to ablate the surface of a chosen target and to force the emission of the target's material in the form of the so-called plasma plume. Upon expanding, the plume reaches the substrate and deposits the material onto its surface. The process parameters like laser fluence, pulse duration, condition of the substrate, determine the properties of the synthesized coating. The adequate choice of the parameters allows for the coating to retain the characteristics of the target, like its stoichiometry and structure.

The PLD deposition was carried out in the vacuum chamber at the base pressure of $10^{-6}$ mbar, at room temperature. The laser used was the Q-switched Nd:YAG, operating at $1064 \mathrm{~nm}$ with pulse duration $10 \mathrm{~ns}$, repetition rate $10 \mathrm{~Hz}$ and fluence $15 \mathrm{~J} / \mathrm{cm}^{2}$.

The target used in the process was a melt-derived bioactive 42S5.2 Na-K glass of composition: $42.34 \mathrm{wt} \%$ $\mathrm{SiO}_{2}, 23.06$ wt $\% \mathrm{CaO}, 11.50$ wt $\% \mathrm{Na}_{2} \mathrm{O}, 17.47$ wt $\% \mathrm{~K}_{2} \mathrm{O}$, $5.63 \mathrm{wt} \% \mathrm{P}_{2} \mathrm{O}_{5} ; \mathrm{Ca} / \mathrm{P}$ molar ratio equal to 5.18. The substrate was a chemically etched, polycrystalline titanium plate. Ti was chosen as, together with its alloys, it is considered to be well tolerated by living organisms and widely used as a material for implants. The targetsubstrate separation was $4 \mathrm{~cm}$.

The morphology of the synthesized coatings was examined with the Raman spectroscopy technique. The bonding configuration of the film and the target was determined as the method is well suited for studies of the $\mathrm{Si}-\mathrm{O}$ groups in glass. The spectra were collected using high resolution, confocal Raman micro-spectrometer Almega XR of Thermo Electron Corp., equipped with the CCD camera for a position sensitive detector. The chosen laser beam wavelength was $532 \mathrm{~nm}$, objective magnification $50 \times$, and a pinhole aperture $25 \mu \mathrm{m}$. The spatial and spectral resolution were $1 \mu \mathrm{m}$ and $2 \mathrm{~cm}^{-1}$, respectively.

\section{Results and discussion}

The PLD-deposited material covered evenly the Ti substrate, however some round particles, a kind of droplets, a few micrometers in diameter, also formed on the coating's surface. This is rather typical of the PLD process and often reported in the literature e.g. [6, 7]. Both the flat layer and the droplets were examined with the Raman micro-spectroscopy and their spectra compared with that of the glass target, Fig. 1.

The major features of the Raman spectra of the silicate glasses are the bands associated with asymmetric and symmetric stretching vibrations of the silica network in the region $850-1200 \mathrm{~cm}^{-1}$ and the bands assigned to the bending and rocking modes around and below $600 \mathrm{~cm}^{-1}$. The former region is of the main interest as it provides information on the glass-network connectivity and, related to it, material's bioactivity.

The distribution and intensity of the $Q^{n}$ components are related to the contribution of $\mathrm{Si}_{x} \mathrm{O}_{y}$ units in the structure and thus to the glass properties. To find these components we deconvoluted the spectrum in the spectral region $700-1300 \mathrm{~cm}^{-1}$ using curve-fitting procedure. The

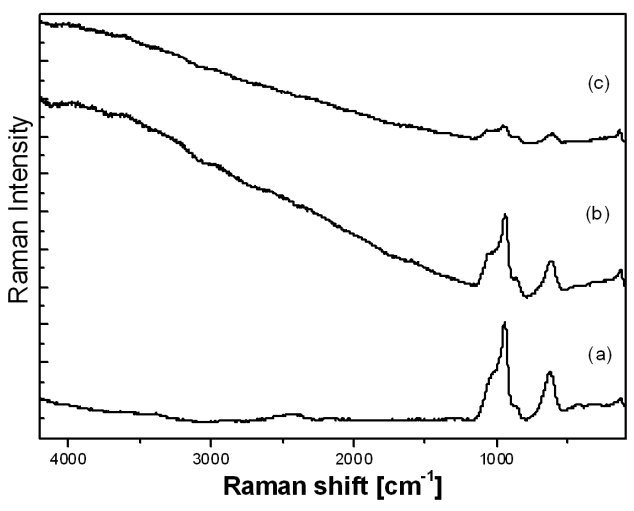

Fig. 1. Raman spectra of the glass target $(a)$ and the coating $(b, c)$. The spectrum $(b)$ was obtained for round particles on the coating's surface.

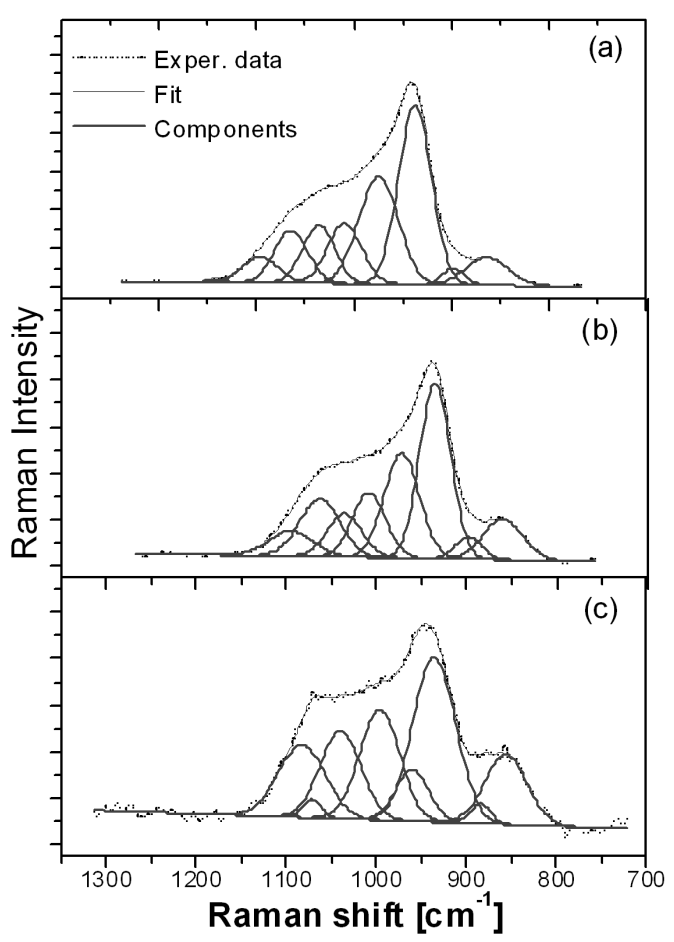

Fig. 2. Components of $\mathrm{Si}-\mathrm{O}-\mathrm{Si}$ stretching-region envelope of the Raman spectra, resolved by the curve-fitting procedure: (a) bioglass target, (b) particles on the surface of the PLD-deposited bioglass coating, and (c) bioglass coating.

Gaussian shape line was chosen as the most adequate for this amorphous, disordered structure. The spectra were baseline corrected prior to the procedure. The results are presented in Fig. 2 and in Tables I and II. 
TABLE I

Raman band positions extracted from spectra deconvolution.

\begin{tabular}{c|c|c|c}
\hline \hline Bioglass-target & Bioglass coating-particles & Bioglass coating & Assignment \\
\hline \multicolumn{2}{c|}{ Raman shift $\left[\mathrm{cm}^{-1}\right]$} \\
864 & 860 & 856 & $Q^{0}$ \\
898 & 898 & 884 & $Q^{1}$ \\
937 & 935 & 939 & $Q^{2}$ \\
974 & 972 & 976 & P-O-P stretching \\
1010 & 1008 & 1009 & O-P-O stretching of $\mathrm{P}_{2} \mathrm{O}_{5}$ \\
1036 & 1035 & 1040 & $\mathrm{BO}$ in all $\mathrm{SiO}_{4}$ units \\
1064 & 1063 & 1071 & $Q^{4} \mathrm{Si}-\mathrm{O}-\mathrm{Si}=120^{\circ}$ \\
1095 & 1095 & 1091 & $Q^{3}$ \\
1140 & - & - & $Q^{4} \mathrm{Si}-\mathrm{O}-\mathrm{Si}=160^{\circ}$
\end{tabular}

TABLE II

Relative abundance of the $Q^{n}$ species estimated from the integrated intensities of the respective Raman bands.

\begin{tabular}{c|c|c|c}
\hline \hline$Q^{n}$ & $\begin{array}{c}\text { Bioglass- } \\
\text {-target }\end{array}$ & $\begin{array}{c}\text { Bioglass coating- } \\
\text { droplets }\end{array}$ & $\begin{array}{c}\text { Bioglass } \\
\text { coating }\end{array}$ \\
\hline$Q^{0}$ & 0.14 & 0.18 & 0.21 \\
$Q^{1}$ & 0.05 & 0.07 & 0.03 \\
$Q^{2}$ & 0.70 & 0.62 & 0.51 \\
$Q^{3}$ & 0.11 & 0.13 & 0.25
\end{tabular}

The ratio of non-bridging oxygens to bridging oxygens, $\mathrm{Si}-\mathrm{O}-\mathrm{NBO} / \mathrm{Si}-\mathrm{O}-\mathrm{Si}$, calculated as the sum of $Q^{0}, Q^{1}$, $Q^{2}, Q^{3}$ contributions over the number of BO contributions $\left(\mathrm{BO}+\mathrm{Q}^{4}\right)$ was found to be 2.3 , for both the target and droplets and 3.5 for the amorphous coating. This points to the higher depolymerization of the silica network in the PLD glass coating with respect to the target. This result is contrary to that obtained by Zhao et al. [7] who reported the ratio to decline in the coating as compared with the target. In the droplets we found the ratio to be the same as in the target, possibly because some of the $\mathrm{Si}-\mathrm{O}-\mathrm{Si}$ were restored due to melting during ablation process or the droplets were in fact bigger particulates ablated from the target and deposited onto the substrate without losing their integrity.

The resolution of the stretching envelope into components revealed that all $Q^{n}$ structural units were present in our glass, apart from bands assigned to $\mathrm{P}-\mathrm{O}$ bonding, both in the target and the coating. Following [10] we assess the relative abundance among the $Q^{0}, Q^{1}, Q^{2}, Q^{3}$ species (corresponding to isolated $\mathrm{SiO}_{4}, \mathrm{Si}_{2} \mathrm{O}_{7}^{6-}, \mathrm{Si}_{2} \mathrm{O}_{6}^{4-}$, $\mathrm{Si}_{2} \mathrm{O}_{5}^{2-}$, respectively) by determining the contributions of the integrated intensities of the respective Raman bands (Table II). It can be seen that the structure is dominated by the $Q^{2}$ units in both the target and PLD-deposited coating. The $Q^{2}$ units represent chains of metasilicates $\left(\mathrm{Si}_{2} \mathrm{O}_{6}^{4-}\right)$ and high abundance of this species is characteristic of highly bioactive glasses. The fraction of the $Q^{2}$ units is only slightly smaller for the PLD coating than that for the target (the $Q^{0}$ and $Q^{3}$ are higher instead) but it is still decidedly dominant among the other $Q^{n}$ species. From this it can be inferred that the glass formed in the PLD process retained the bioactive properties of the target glass.

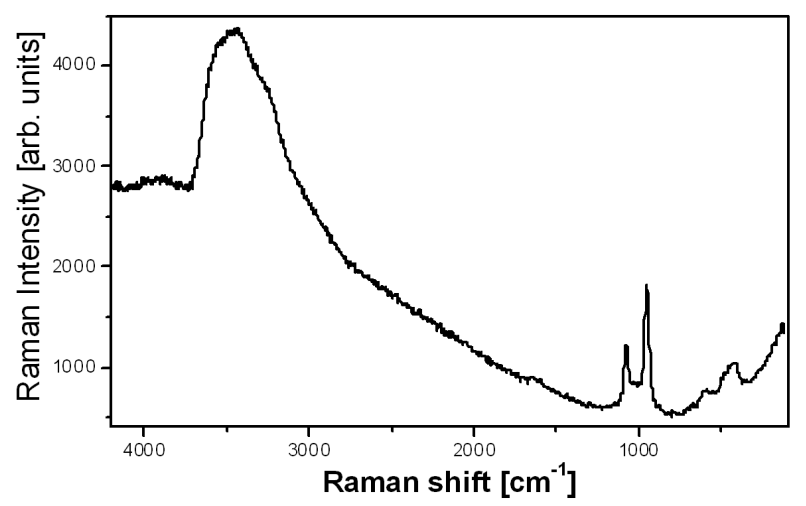

Fig. 3. Raman spectrum of hydroxyapatite formed on the glass coating soaked in SBF (as measured, no baseline correction).

The bioactivity test was carried out in vitro by soaking the sample in the commercial simulated body fluid (SBF) for a few days at room temperature and examining the sample surface with the Raman micro-spectroscopy. The representative Raman spectrum is shown in Fig. 3 and was found to be that of hydroxyapatite (stoichiometric formula $\left(\mathrm{Ca}_{10}\left(\mathrm{PO}_{4}\right)_{6}(\mathrm{OH})_{2}\right)$ (see e.g. [11, 12]). It contains two relatively strong bands at $952 \mathrm{~cm}^{-1}\left(\mathrm{PO}_{4} \nu_{1}\right.$ vibration) and $1076 \mathrm{~cm}^{-1}\left(\mathrm{PO}_{4} \nu_{3}\right.$ vibration) and two phosphate bands in the range 400 to $500 \mathrm{~cm}^{-1}$ (due to $\nu_{2}$ ) and 550 to $650 \mathrm{~cm}^{-1}\left(\nu_{4}\right)$. The broad band around $3500 \mathrm{~cm}^{-1}$ on the fluorescent background is due to $\mathrm{OH}$ stretching. No glass lines are present in the spectrum indicating that the thickness of the apatite layer is greater than $2 \mu \mathrm{m}$ (confocal depth resolution of the used microscope).

The presence of the apatite layer on the surface of the coating proved that the desired chemical reaction with 
the SBF did take place and that the coating preserved the bioactive properties.

\section{Conclusions}

In the reported PLD process, the bioactive glass which was transferred from the target to the Ti substrate retained its bioactive properties. The proportion of non-bridging oxygens to bridging oxygens, $\mathrm{Si}-\mathrm{O}-\mathrm{NBO} / \mathrm{Si}-$ $\mathrm{O}-\mathrm{Si}$ ratio, considered to be the factor determining the bioactivity of the glass has risen in the coating, indicating that the coating's chemical activity may be even higher than that of the target. All $Q^{n}$ components (i.e. $Q^{0}, Q^{1}$, $Q^{2}, Q^{3}$, and $Q^{4}$ ) were found to be present in our samples, i.e. in the glass target, the coating and the droplets, the $Q^{1}$ being very small and $Q^{2}$ the most significant in all. Some redistribution of the $\mathrm{Si}_{x} \mathrm{O}_{y}$ species occurred in the coating with respect to the target, namely the abundance of the $Q^{0}$ and $Q^{3}$ components (corresponding to 4 and 1 NBO, respectively) increased at the expense of $Q^{2}$ component (2 NBOs). Thus there were more isolated $\mathrm{SiO}_{4}$ tetrahedra and $\mathrm{Si}_{2} \mathrm{O}_{5}^{2-}$ units in the coating. The contribution of the $Q^{2}$ unit representing the metasilicates, or $\mathrm{Si}_{2} \mathrm{O}_{6}^{4-}$ chains, remains the most significant in the coating, as it is in the target and the droplets. The results point to the high degree of depolymerization of the silica network in the glass coating and high abundance of non-bridging oxygens resulting in high bioactivity of the glass. The findings were further supported by the results of the in vitro bioactivity test.

\section{Acknowledgments}

We thank Dr. M. Ciecińska from AGH University of Science and Technology for providing the bioactive-glass target.
The work was supported by the project no. SPO WKP-1/1.4.3/1/2004/90/90/186, "Nanotechnology of complex coatings for special medical and industrial application".

\section{References}

[1] T. Kokubo, Acta Mater. 46, 2519 (1998).

[2] T. Kokubo, H. Kim, M. Kawashita, Biomaterials 24, 2161 (2003).

[3] A.C. Popescu, F. Sima, L. Duta, C. Popescu, I.N. Mihailescu, D. Capitanu, R. Mustata, L.E. Sima, S.M. Petrescu, D. Janackovic, Appl. Surf. Sci. 255 5486 (2009)

[4] O. Peitl, E.D. Zanotto, L.L. Hench, J. Non-Cryst. Solids 292, 115 (2001).

[5] A. Tilocca, Proc. R. Soc. A 465, 1003 (2009).

[6] I.W. Boyd, Ceram. Int. 22, 429 (1996).

[7] Y. Zhao, M. Song, C. Chen. J. Liu, Appl. Surf. Sci. 254, 6897 (2008).

[8] S. Liste, J. Serra, P. González, J.P. Borrajo, S. Chiussi, B. León, M. Pérez-Amor, Thin Solid Films 453-454, 224 (2004).

[9] L.L. Hench, R.J. Splinter, W.C. Allen, T.K. Greenlee, J. Biomed. Mater. Res. Symp. (Part 1) 4, 117 (1971).

[10] C.C. Lin, L.-C. Huang, P. Shen, J. Non-Cryst. Solids 351, 3195 (2005).

[11] H. Tsuda, J. Arends, J. Dent. Res. 73, 1703 (1994).

[12] G. Penel, G. Leroy, C. Rey, B. Sombret, J.P. Huvenne, E. Bres, J. Mater. Sci. - Mater. Med. 8, 271 (1997). 